SUBJECT AREAS:

ATHEROSCLEROSIS

METABOLIC SYNDROME

Received

23 January 2014

Accepted

17 April 2014

Published

8 May 2014

Correspondence and requests for materials should be addressed to

N.M.

(norikazu_maeda@ endmet.med.osaka-u. ac.jp) or Y.N.

lynakamura@anatl. med.osaka-u.ac.jp)

* These authors contributed equally to this work.

\section{Adiponectin protein in Vasculature of Normal and Atherosclerotic mice}

\author{
Takuya Mori' *, Yoshihisa Koyama²*, Norikazu Maeda', Yukiko Nakamura², Yuya Fujishima' \\ Keisuke Matsuda' ${ }^{1}$ Tohru Funahashi ${ }^{1,3}$, Shoichi Shimada ${ }^{2}$ \& lichiro Shimomura ${ }^{1}$
}

'Department of Metabolic Medicine, Graduate School of Medicine, Osaka University, Suita, Osaka, Japan 565-0871,
2Department of Neuroscience and Cell Biology, Graduate School of Medicine, Osaka University, Suita, Osaka, Japan 565-0871,
3Department of Metabolism and Atherosclerosis, Graduate School of Medicine, Osaka University, Suita, Osaka, Japan 565-0871.

Adiponectin, adipose-specific secretory protein, abundantly circulates in bloodstream and its concentration is around 1000-fold higher than that of other cytokines and hormones. Hypoadiponectinemia is a risk factor for atherosclerosis. There is little or no information on ultrastructural localization of adiponectin in the vasculature. Herein we investigated the localization of vascular adiponectin in the aorta using the immunoelectron microscopic technique. In wild-type (WT) mice, adiponectin was mainly detected on the luminal surface membrane of endothelial cells (ECs) and also found intracellularly in the endocytic vesicles of ECs. In the atherosclerotic lesions of apolipoprotein E-knockout (ApoE-KO) mice, adiponectin was detected in ECs, on the cell surface membrane of synthetic smooth muscle cells, and on the surface of monocytes adherent to ECs. Changes in adiponectin localization within the wall of the aorta may provide novel insight into the pathogenesis of atherosclerosis.

therosclerosis is a common disease in the industrial countries and sometimes develops into fatal diseases such as myocardial infarction and stroke. Understanding the pathogenesis of atherosclerosis should allow the design of new preventive and therapeutic paradigms for cardiovascular diseases. The classical Virchow-Aschoff hypothesis states that lipids in atherosclerotic arterial wall lesions originate from the bloodstream $^{1}$. In this regard, various circulating proteins, such as low-density lipoprotein (LDL), fibrinogen, immunoglobulins, and albumin, which abundantly circulate in the blood and range in concentration from $10^{3}$ to $10^{4} \mu \mathrm{g} / \mathrm{mL}$, are present in atherosclerotic lesions $\mathrm{s}^{2-5}$ and can be detected in atherosclerotic lesions ${ }^{2}$. Furthermore, the process of atherosclerogenesis involves increased endothelial permeability in the damaged vasculature, several immune cells (e.g., monocytes/macrophages and T-lymphocytes), and molecules [e.g., vascular adhesion molecule-1 (VCAM-1), monocyte chemoattractant protein-1 (MCP-1), platelet-derived growth factor (PDGF), and tumor necrosis factor- $\alpha($ TNF- $\alpha)]^{6}$. These factors interactively cause and accelerate local vascular inflammation in atherosclerotic lesions, consisting of endothelial cells, macrophages, T-lymphocytes, and smooth muscle cells ${ }^{7,8}$.

Accumulating evidence indicates that visceral fat obesity is located upstream of the metabolic syndrome and atherosclerosis ${ }^{9,10}$. In the Human Body Map project ${ }^{11}$, our group demonstrated systematically for the first time that adipose tissue serves as not only an energy storage organ but also an endocrine organ ${ }^{12}$. During the completion of the project, our group identified adiponectin among human adipose tissue $\mathrm{cDNAs}^{13}$. During the same period, gelatin-binding protein of $28 \mathrm{kDa}$ (GBP28) was discovered in human plasma and later turned out to be the same protein of adiponectin ${ }^{14}$. Adipocyte complement-related protein of $30 \mathrm{kDa}$ (ACRP30) and AdipoQ, which are mouse homologues of adiponectin, were also independently identified at that time ${ }^{15,16}$. The plasma levels of adipose-derived bioactive substances, which we named "adipocytokines" [e.g., plasminogen activator inhibitor-1 (PAI-1), MCP-1, TNF- $\alpha$ ], generally increase with weight gain. Interestingly, plasma adiponectin levels correlate negatively with body mass index (BMI), although adiponectin is specifically secreted by adipocytes ${ }^{17}$. In normal adults, adiponectin blood level ranges from 1 to $30 \mu \mathrm{g} / \mathrm{mL}$, which is around 1000 -fold higher than the serum concentrations of other cytokines and hormones, ${ }^{9,10}$. Taking into consideration the high serum concentrations of adiponectin, the biological functions of adiponectin may differ from typical cytokines and hormones, but the significance of hyperadiponectinemia is not fully understood. 
(a)

(b) WT
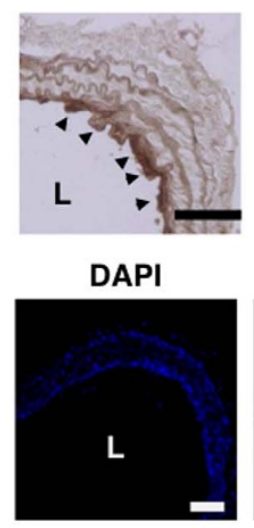

WT
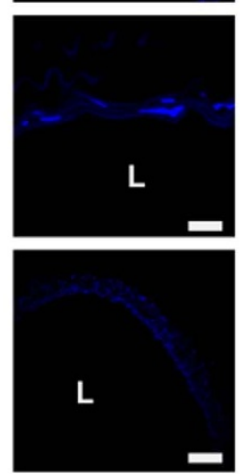

(c)

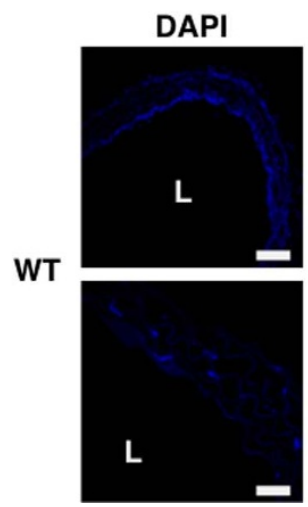

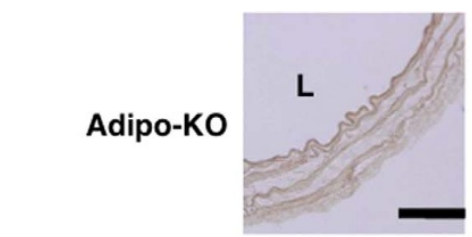

Adiponectin
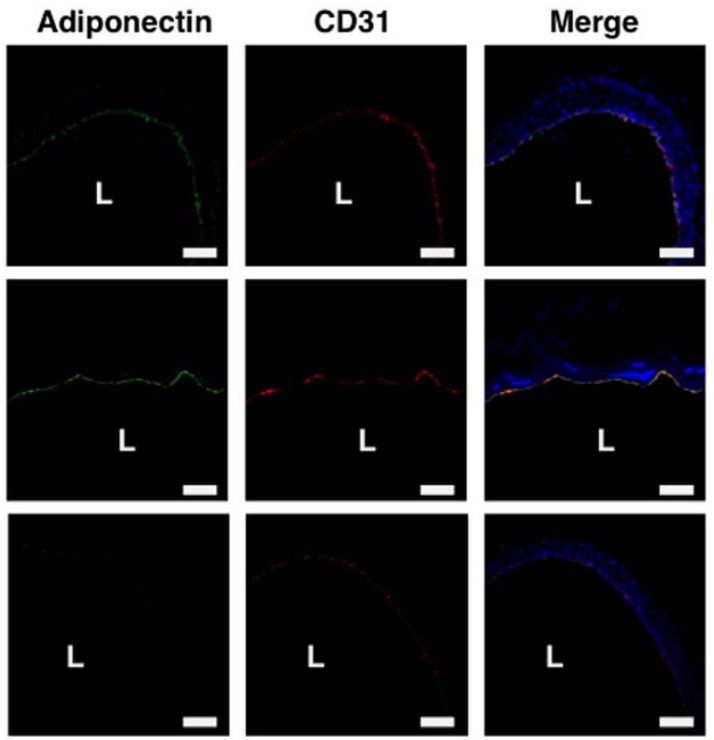

Adiponectin
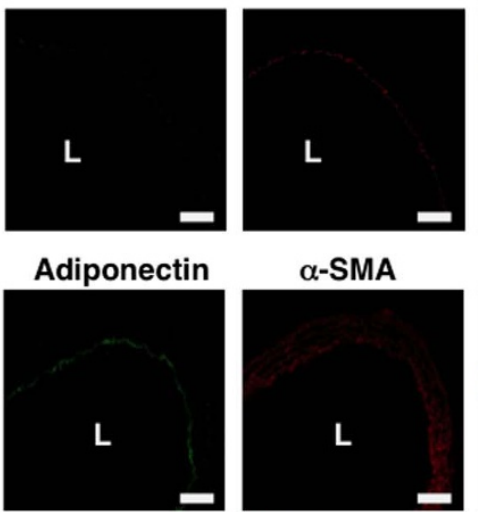

$\alpha$-SMA
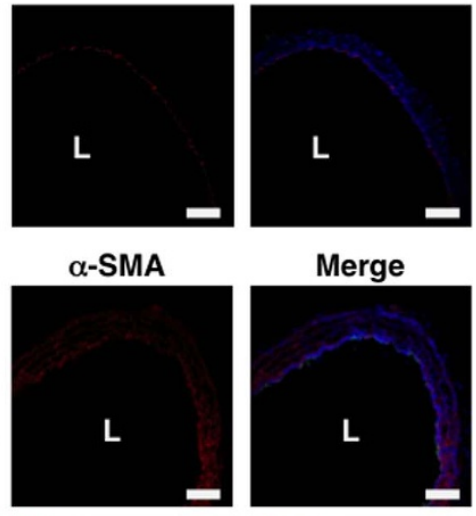

Merge

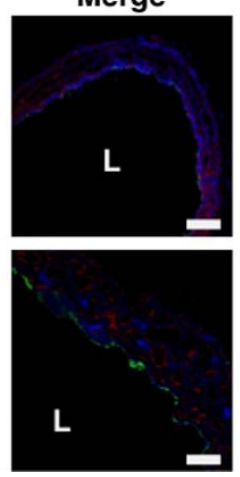

Figure 1 Localization of adiponectin in normal aorta. (a) Immunohistochemical staining for adiponectin in sections of the aorta from WT mice (left) and adiponectin knockout (Adipo-KO) mice (right), visualized with 3,3'-diaminobenzidine tetrahydrochloride (DAB, brown). Arrowheads indicate adiponectin-positive staining. Scale bar $=100 \mu \mathrm{m}$. (b) Immunofluorescence for adiponectin (green) and CD31 (Red) with DAPI-stained nuclei (blue) in WT (top and middle panels) and Adipo-KO (bottom panels) mice. Scale bar $=100 \mu \mathrm{m}$ (top and bottom panels) and $20 \mu \mathrm{m}$ (middle panels).

(c) Immunofluorescence for adiponectin (green) and $\alpha$-smooth muscle actin ( $\alpha$-SMA, red) with DAPI-stained nuclei (blue) in WT mice. Scale bar $=100 \mu \mathrm{m}$ (top panels) and $20 \mu \mathrm{m}$ (bottom panels). In b and c, panels on the right side show merged images. L, lumen.

Previous studies showed that hypoadiponectinemia is associated with lifestyle-related diseases such as coronary artery diseases, type 2 diabetes, and metabolic syndrome ${ }^{18-22}$. Studies using adiponectin knockout (Adipo-KO) mice and overexpression of adiponectin, have confirmed that the physiological functions of adiponectin include anti-diabetic ${ }^{23-25}$, anti-atherogenic ${ }^{26,27}$, anti-inflammatory ${ }^{28}$, and anti-fibrotic $^{29,30}$ activities. Furthermore, in vitro experiments using vascular component cells indicated that adiponectin also has antiatherogenic activity during the process of atherosclerogenesis ${ }^{31-34}$. Using confocal laser scanning microscope, our group showed recently the existence of adiponectin protein in the intima layer of the mouse aortic endothelium ${ }^{35}$. However, the precise localization of adiponectin in the normal vascular endothelial cells and atherosclerotic lesions remains uncertain. The present study investigated the precise localization of adiponectin protein the vasculature by immunoelectron microscopy.

\section{Results}

Immunohistochemical detection of adiponectin in normal aorta. To map the arterial wall for adiponectin protein, we used the thoracic ascending aorta of wild-type (WT) mice and immunohistochemistry. Adiponectin protein was detected in the intima layer (Fig. 1a, arrowheads in left panel), in agreement with our recent study using western blotting ${ }^{35}$. Immunofluorescence staining using goat IgG for adiponectin showed no adiponectin in the aorta (Supplementary Fig. S1a). In addition, the antibody absorption test demonstrated that adiponectin signal was abolished when anti-adiponectin antibody was co-incubated with a 20 -fold molar excess of the synthetic peptide of adiponectin (Supplementary Fig. S1b). Adiponectin signal was not detected in the aortic intima (Fig. 1a, right panel) and endothelium (Fig. 1b, bottom panels) of Adipo-KO mice. These results indicate that the adiponectin-positive signal was a specific immunoreaction for adiponectin. For precise localization of adipo- 
(a)

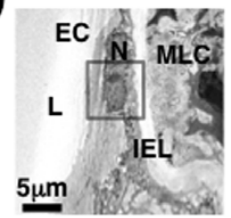

(c)

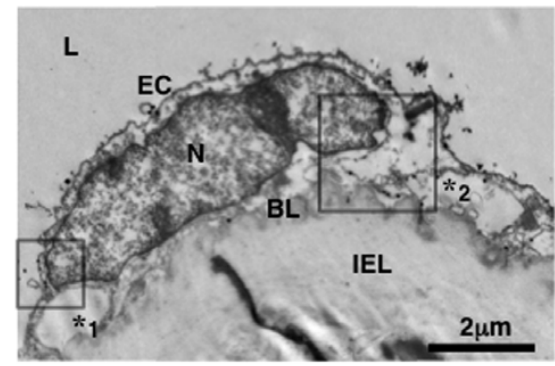

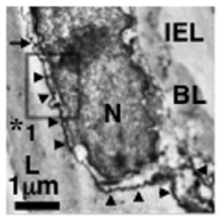

(b)

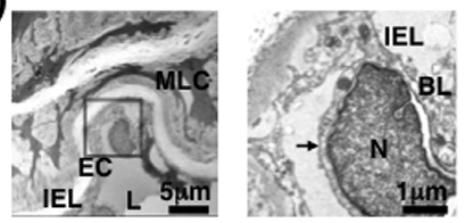

(d)

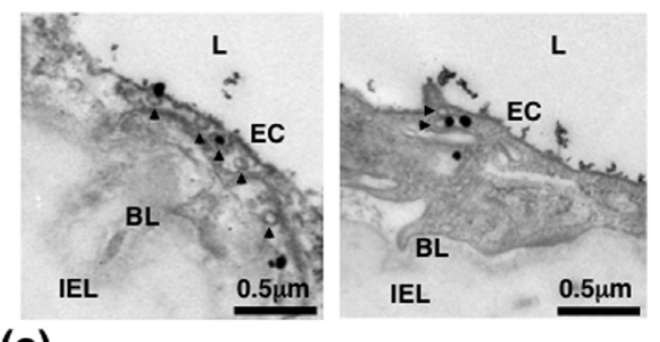

(e)

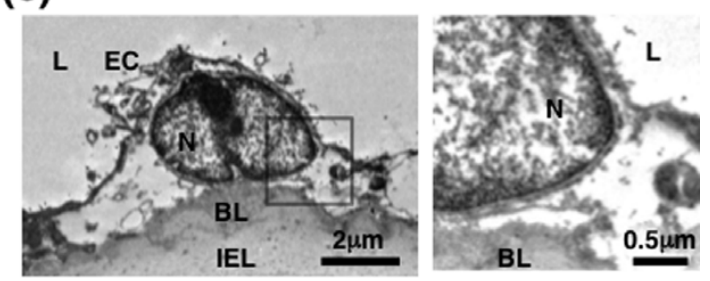

Figure $2 \mid$ Immunoelectron microscopic localization of adiponectin in normal aorta. (a, b) Immunoelectron micrographs using a pre-embedding immunoperoxidase technique for adiponectin in sections of the aorta from WT mice (a) and adiponectin knockout (Adipo-KO) mice (b). Middle panel (a) and right panel (b): higher magnifications of the region outlined in the left panels, respectively. Right panel of (a): higher magnification of the *1 region outlined in the middle panel. Arrows in (a) and (b): plasma membrane of endothelial cells (ECs). Arrowheads in (a): reaction products of 3,3'diaminobenzidine tetrahydrochloride (DAB, black dots). (c, d) Immunoelectron micrographs using a pre-embedding immunogold labeling technique for adiponectin in sections of the aorta from WT mice. Lower two panels in (c): higher magnifications of the * 1 and *2 regions outlined in the top panel in (c), respectively. Arrows in (c): pits on the plasma membrane of ECs. Arrowheads in (d): endocytic vesicles in ECs. (e) Immunoelectron micrographs for adiponectin using the pre-embedding immunogold labeling technique in sections of the aorta from Adipo-KO mice. Right panel of (e): higher magnification of the region outlined in left panel. L, lumen; EC, endothelial cell; N, nucleus; IEL, internal elastic lamina; BL, basal lamina; MLC, myofibroblast-like cell.

nectin, double-immunofluorescence staining was performed for adiponectin and CD31 (endothelial cell marker), and $\alpha$-SMA [smooth muscle cell (SMC) marker]. Adiponectin was co-stained with CD31 (Fig. 1b, top panels), but not with $\alpha$-SMA (Fig. 1c). Examination under higher magnification showed colocalization of adiponectin with CD31 (Fig. 1b, middle panels), indicating that adiponectin is localized mainly in endothelial cells (ECs) in the aorta of WT mice.

Immunoelectron microscopic analysis of adiponectin localization in normal aorta. For ultrastructural localization of adiponectin, immunoelectron microscopy was performed in thoracic ascending aorta of WT mice. Sites immunoreactive to adiponectin and visualized with $\mathrm{DAB}$ were observed on the luminal and basal plasma membrane of ECs (Fig. 2a). Furthermore, immunoreactive sites of adiponectin were also observed in endocytic vesicles (Fig. 2a, arrowheads in right panel). On the other hand, no immunoreactive sites were observed on the plasma membrane of ECs in the aortic intima of Adipo-KO mice used as negative control (Fig. 2b). Consistent with light microscopic analyses, there were no DAB reaction products for adiponectin on other vascular component cells, such as the myofibroblast-like cells (MLCs) and SMC (Supplementary Fig. S2).

The presence of adiponectin in endocytic vesicles was confirmed further by the pre-embedding immunogold labeling technique. Similar to the observation in immunoelectron microscopy using the pre-embedding immunoperoxidase technique, adiponectin was detected on the luminal and basal plasma membrane of ECs (Fig. 2c).
Interestingly, the labeled adiponectin on luminal plasma membrane was also observed in pit-like structures known as sites of endocytosis (Fig. 2c, arrows). Adiponectin-positive immunogold particles were identified in endocytic vesicles, thus confirming the observation using DAB (Fig. 2d, arrowheads). Adiponectin was not detected on SMCs (Supplementary Fig. S3a), confirming the findings of light and $\mathrm{DAB}$ immunoelectron microscopy. The immunoreactive signal was specific for adiponectin, because adiponectin signal was detected in perivascular adipocytes in positive control sections (Supplementary Fig. S3b) and immunogold-labeled adiponectin was not detected in ECs in the aortic intima of Adipo-KO mice (negative control) (Fig. 2e). These results indicate that adiponectin is mainly localized in ECs and partly in endocytic vesicles in normal mice.

Immunohistochemical detection of adiponectin in atherosclerotic lesions. To determine the localization of adiponectin protein under pathological state, we examined atherosclerotic lesions on the thoracic ascending aorta of ApoE-KO mice fed western diet (WTD) for 12 weeks. Hematoxylin and eosin (H\&E)- and Oil red $\mathrm{O}$-stained sections confirmed the presence of atherosclerosis in these ApoE-KO mice (Fig. 3a). As shown in Supplementary Figure S4, adiponectin protein level was significantly higher in aortas of ApoE-KO mice (495 $\pm 35 \mathrm{ng} / \mathrm{mg}$ protein) than in WT mice (304 $\pm 19 \mathrm{ng} / \mathrm{mg}$ protein). Next, we delineated the difference in adiponectin localization in ApoE-KO mice relative to WT mice. Immunohistochemical staining with $\mathrm{DAB}$ showed adiponectin staining in the endothelium of both ApoE-KO mice (Fig. 3b) and WT mice (Fig. 1a). However, unlike WT mice, staining for 
(a)
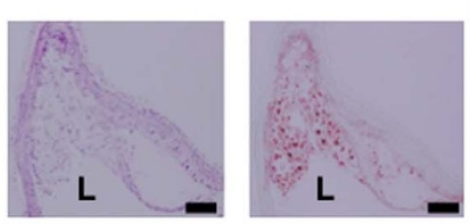

(b)
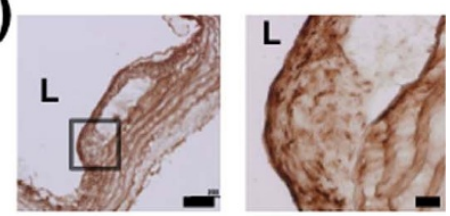

(c)
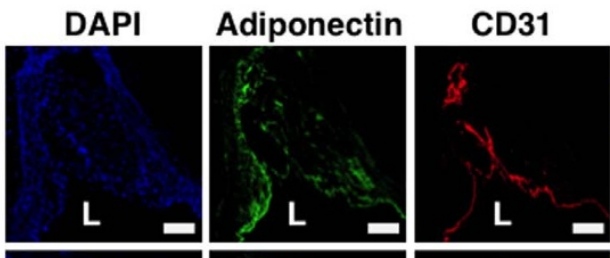

\section{Merge}
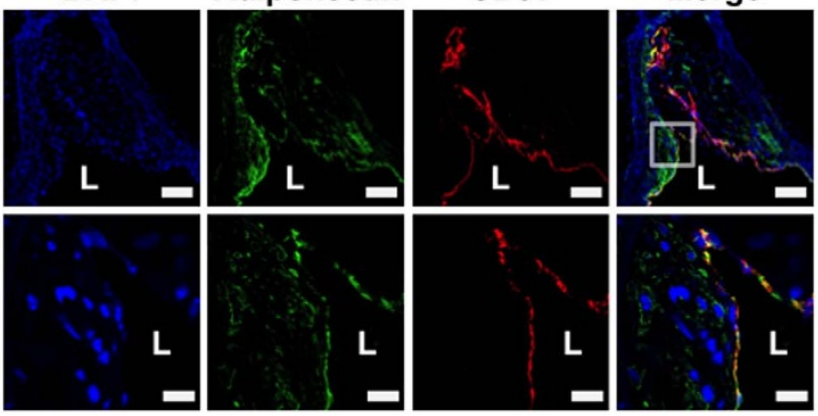

(d)
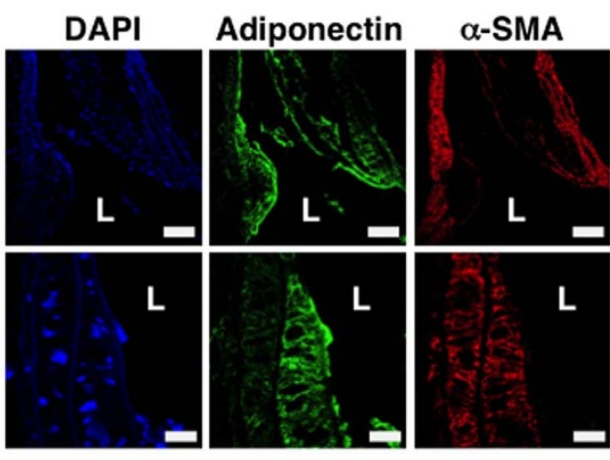

Merge
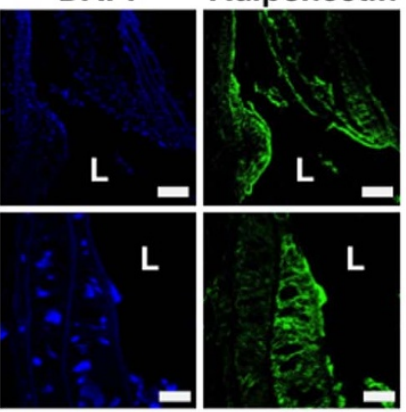

(e)
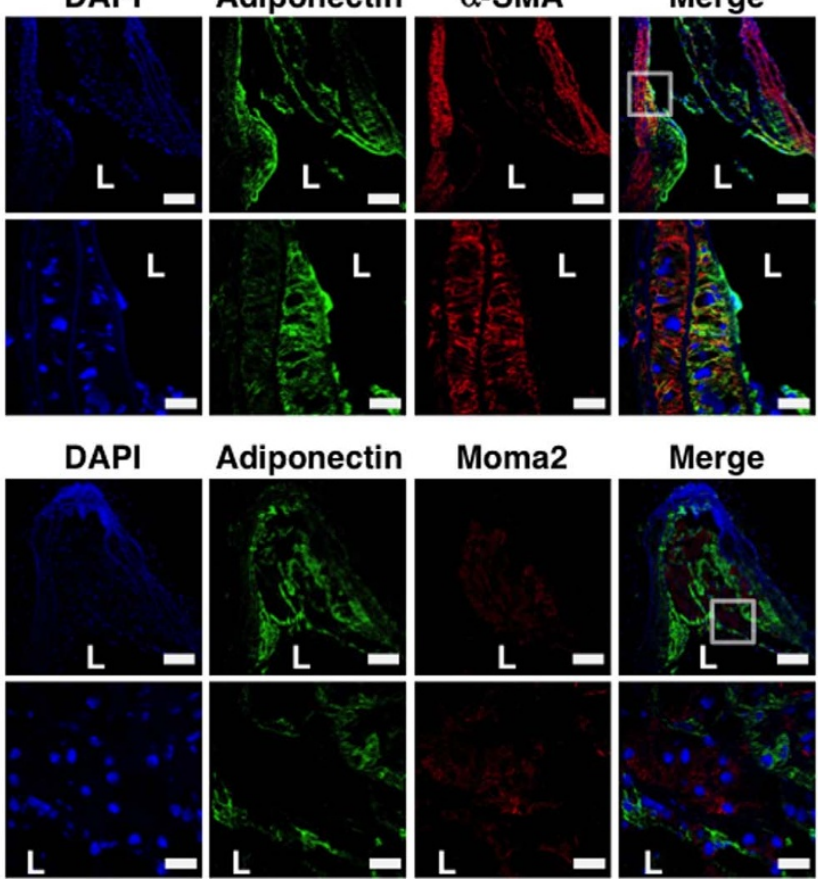

Merge

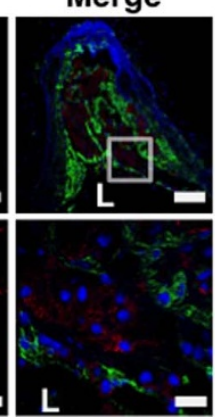

Figure $3 \mid$ Immunohistochemical staining of adiponectin in atherosclerotic lesions. Apolipoprotein E knockout (ApoE-KO) mice were fed high-fat atherogenic Western diet (WTD) from 8 weeks of age and analyzed at 20 weeks of age. (a) Representative histological analysis of the aorta of ApoE-KO mice. Sections were stained with hematoxylin and eosin (H\&E) (left panel) and Oil red O (right panel). Scale bar $=100 \mu \mathrm{m}$. (b) Immunohistochemical staining for adiponectin in sections of the aorta from ApoE-KO mice. Adiponectin was visualized with 3,3'-diaminobenzidine tetrahydrochloride (DAB, brown). Right panel: higher magnification of the region outlined in left panel. Scale bar $=100 \mu \mathrm{m}$ (left panel) and $20 \mu \mathrm{m}$ (right panel).

(c) Immunofluorescence for adiponectin (green) and CD31 (red) in sections of the aorta from ApoE-KO mice. (d) Immunofluorescence for adiponectin (green) and $\alpha$-smooth muscle actin ( $\alpha$-SMA, red) in sections of the aorta from ApoE-KO mice. (e) Immunofluorescence for adiponectin (green) and moma-2 (red) in sections of the aorta from ApoE-KO mice. In c to e, cell nuclei were counterstained with DAPI (blue). Panels on the right side in c to e show the merged images. In c to e, scale bar $=100 \mu \mathrm{m}$ (top panels) and $20 \mu \mathrm{m}$ (bottom panels). L, lumen.

adiponectin was also noted in the subendothelial space in the atherosclerotic lesions of ApoE-KO mice (Fig. 3b).

Double immunofluorescence staining was therefore performed to determine the precise localization of adiponectin in the atherosclerotic lesions (Fig. 3c-e). Similar to WT mice, adiponectin co-stained with CD31 in ApoE-KO mice (Fig. 3c), indicating its presence in ECs. Higher magnification showed stronger colocalization of adiponectin with CD31 in ApoE-KO mice compared with WT mice (Fig. 3c, bottom panels). Unlike WT mice, adiponectin was co-stained with $\alpha$-SMA in the atherosclerotic lesions (Fig. 3d), suggesting localization of adiponectin on SMCs of ApoE-KO mice. The overlap of adiponectin staining with $\alpha$-SMA was mainly observed in the subendothelium at the atherosclerotic cap and shoulder of the plaque, but was not detected in basal side of the media layer (Fig. 3d, bottom panels). Previous studies described macrophage invasion at lesion site and thus we examined the expression of adiponectin in macrophages using immunofluorescence staining for Moma-2, a marker of macrophages. Adiponectin did not co-stain with Moma-2, indicating that it is not localized in macrophages (Fig. 3e). Immunofluorescence staining with goat IgG for adiponectin (Supplementary Fig. S5a) and antibody absorption test (Supplementary Fig. S5b) confirmed that the adiponectin-positive signal was a specific immuno-reaction for 
(a)

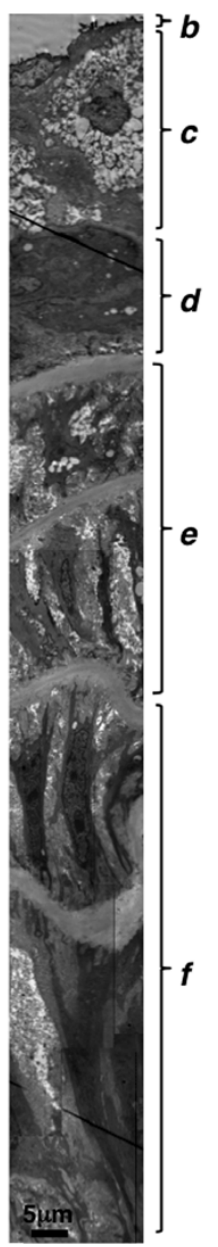

(b)
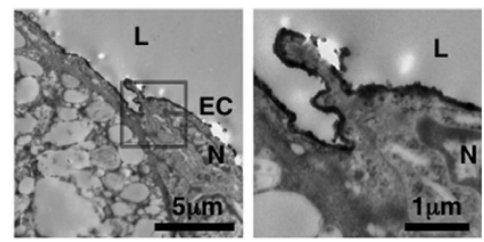

(c)
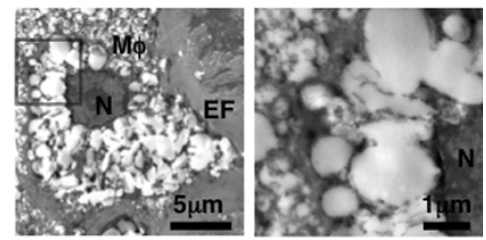

(d)
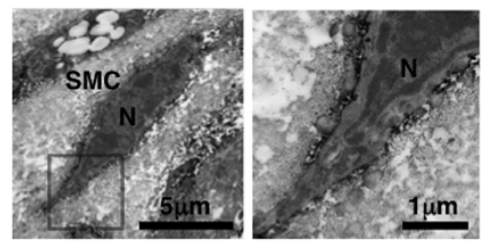

(e)
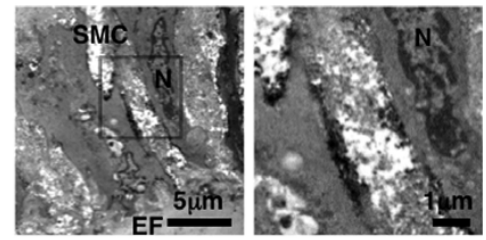

(f)
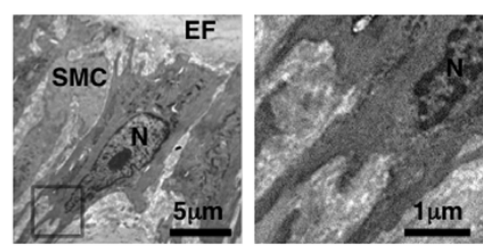

Figure $4 \mid$ Immunoelectron microscopic localization of adiponectin in atherosclerotic lesions. Apolipoprotein E knockout (ApoE-KO) mice were fed high-fat atherogenic Western diet (WTD) diet from 8 weeks of age and analyzed at 20 weeks of age. (a) Overview of immunoelectron micrographs using the pre-embedding immunoperoxidase technique for adiponectin in sections of the aorta from ApoE-KO mice. (b-f) Immunoelectron micrographs for adiponectin in endothelial cells (b), macrophages (c), smooth muscle cells in the intima (d) and in the media (e, f) in sections of the aorta from ApoE-KO mice. Panels on the right side of $b$ to $f$ : higher magnifications of the region outlined in the left panel, respectively. L,

lumen; EC, endothelial cell; M $\phi$, macrophage; SMC, smooth muscle cell; $\mathrm{N}$, nucleus; $\mathrm{EF}$, elastic fiber.

adiponectin in the atherosclerotic lesions of ApoE-KO mice, similar to the normal aorta of WT mice. In addition, immunofluorescence staining with the respective control IgG for CD31, $\alpha$-SMA, and Moma-2 were negative in the aortas of ApoE-KO mice (Supplemental Figure S6a to S6c).

Immunoelectron microscopic analysis of adiponectin localization in atherosclerotic lesions. For precise localization of adiponectin in the atherosclerotic lesions, immunoelectron microscopy using a preembedding immunoperoxidase technique was conducted using thoracic ascending aortas of ApoE-KO mice fed with WTD for 12 weeks. In agreement with the results of light microscopy, adiponectin immunoreactive sites visualized with DAB staining were detected in the endothelium and subendothelial space of the atherosclerotic lesions (Fig. 4a and 4b). Adiponectin signal was mainly observed in the luminal plasma membrane of ECs in atherosclerotic lesions (Fig. 4b), compared with the luminal and basal plasma membranes of ECs in the normal aorta (Fig. 2a). Staining for adiponectin was not

visibly detectable in lipid-loaded macrophages found in the subendothelium (Fig. 4c). Although immunofluorescence showed the presence of adiponectin in SMCs, the reaction products were located in the membrane of SMCs in the intima (Fig. 4d) and media (Fig. 4e) around the atherosclerotic plaque. Although the contractile SMCs make rigid contact with the elastic fibers, such contact looked looser in adiponectin-positive SMCs, which is classified as synthetic/proliferative phenotype. As shown in Fig. 4e, adiponectin was detected in the medial SMCs, which is classified as pre-proliferative phenotype. On the other hand, no immunoreactivity was detected for adiponectin in the contractile SMCs in the media away from the atherosclerotic plaque (Fig. 4f).

We also examined the subcellular localization of adiponectin in the atherosclerotic lesions by immunoelectron microscopy using the pre-embedding immunogold labeling technique. Adiponectin was mainly found in the luminal plasma membrane of ECs on atherosclerotic lesions (Fig. 5a). Careful examination of SMCs in the atherosclerotic lesions by serial immunoelectron micrographs confirmed the presence of adiponectin mainly on the cell surface membrane (Fig. 5b). Furthermore, no adiponectin signal was observed in SMCs of WT and Adipo-KO mice, compared with strong signal in ApoE-KO mice. Quantitative analysis showed that the number of adiponectin particles per cell was $11.0 \pm 3.4(n=5)$ in ApoE-KO mice.

Immunoelectron microscopy using the pre-embedding immunoperoxidase technique detected monocytes adherent to ECs on the atherosclerotic lesions, which were not observed in WT mice. Furthermore, adiponectin immunoreactive products were also found on the surface membrane of these monocytes (Fig. 6). Five adiponectin-positive monocytes attached to ECs were identified by immunoelectron microscopy.

\section{Discussion}

The main findings of the present study were: (1) Adiponectin protein was present mainly on the luminal surface membrane of ECs and to some extent on the basal endothelial membrane. (2) Part of endothelial adiponectin was detected in the endocytic vesicle. (3) In the atherosclerotic lesions of ApoE-KO mice, adiponectin signal was detected not only on ECs but also on SMCs. (4) Adiponectin signal was detected on the surface of monocytes adherent to ECs in atherosclerotic lesions of ApoE-KO mice.

Ross et $\mathrm{al}^{36}$ provided the well-known "response-to-injury" hypothesis of atherosclerosis. According to this hypothesis, ECs are activated by vascular injury such as oxidized low-density lipoprotein (ox-LDL) and mechanical stresses, express endothelial adhesion molecules, and consequently, recruit monocytes into the intima ${ }^{37}$. Using immunoelectron microscopy, the present study confirmed the presence of adiponectin protein on the vascular ECs, adding support to the finding of our recent study ${ }^{35}$. The results also showed that the amount of endothelial adiponectin protein was higher in the atherosclerotic lesion of ApoE-KO mice than in the normal aorta of WT mice (Fig. 2 versus Figs. 4 and 5), although adiponectin was present only in ECs in the non-atherosclerotic lesions of ApoE-KO mice, similar to WT mice (data not shown). Adiponectin is known to suppress any increase in adhesion molecules induced by inflammatory stimuli ${ }^{31,32,35}$. Increased endothelial permeability ${ }^{2}$ may promote accumulation of adiponectin in the atherosclerotic lesion, but the exact molecular mechanism responsible for such accumulation is not clear at this stage.

Adiponectin signal was also detected on the basal membrane of ECs in both WT and ApoE-KO mice (Figs. 2 and 5), though the mechanisms involved in this localization are obscure at present. Two possible pathways could explain the localization of adiponectin on the basal membrane of ECs: (1) Adiponectin crosses ECs from the luminal to basal membrane. (2) Adiponectin permeates through the extracellular space among endothelial cell monolayer and attaches to 
(a)
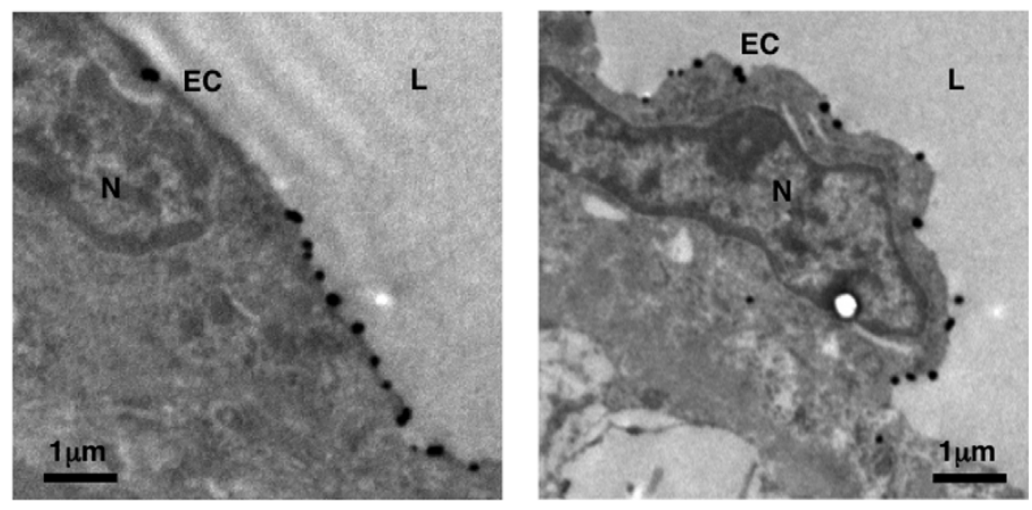

(b)
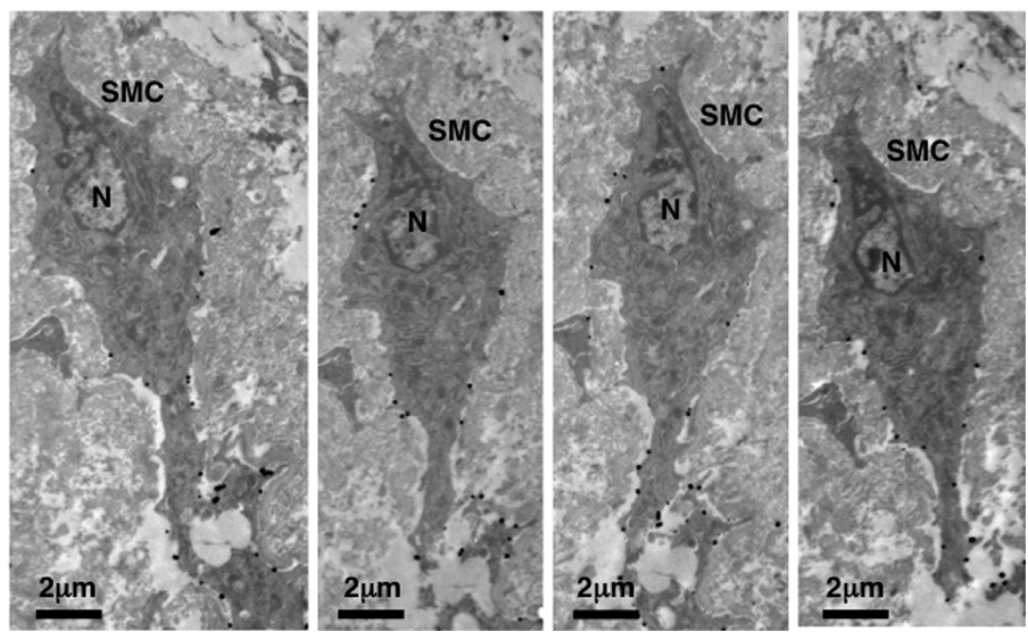

Figure 5 Subcellular localization of adiponectin in atherosclerotic lesions. Apolipoprotein E knockout (ApoE-KO) mice were fed high-fat atherogenic Western diet (WTD) from 8 weeks of age and analyzed at 20 weeks of age. (a) Immunoelectron micrographs using the pre-embedding immunogold labeling technique for adiponectin in endothelial cells in the aortic intima of ApoE-KO mice. (b) Serial immunoelectron micrographs using the preembedding immunogold labeling technique for adiponectin in smooth muscle cells in the intima layer of aorta of ApoE-KO mice. L, lumen; EC, endothelial cells; SMC, smooth muscle cells; N, nucleus.

the basal membrane of ECs. Immunoelectron microscopy showed no adiponectin signal on the intracellular basal side of ECs though such signal was observed in the extracellular space between ECs. These findings suggest that adiponectin permeates through the extracellular space among ECs and reaches the basal endothelial membrane. Further studies are needed to investigate the biological significance of
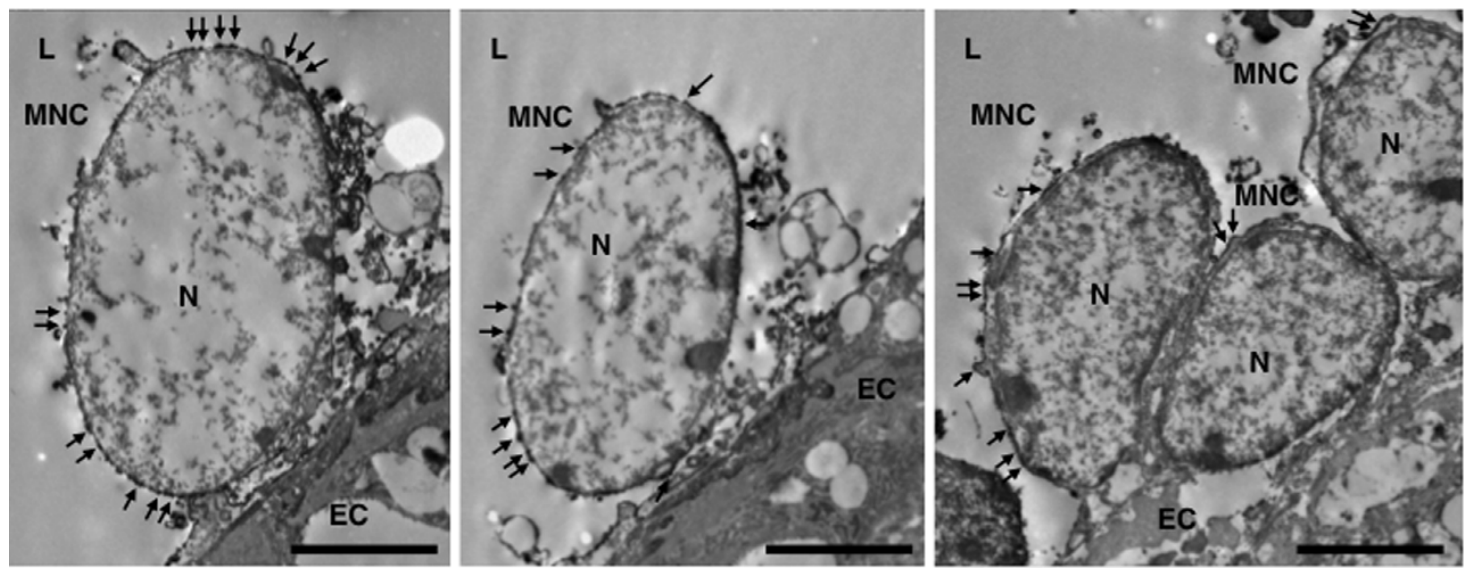

Figure 6 Immunoelectron micrographs for adiponectin at monocytes adhering to endothelial cells in atherosclerotic lesions. Apolipoprotein $\mathrm{E}$ knockout (ApoE-KO) mice were fed high-fat atherogenic Western diet (WTD) from 8 weeks of age and analyzed at 20 weeks of age. Immunoelectron micrographs using the pre-embedding immunoperoxidase technique for adiponectin in aorta of ApoE-KO mice. Arrows: reaction products of 3,3' diaminobenzidine tetrahydrochloride (DAB, black dots). Scale bar $=5 \mu \mathrm{m}$. L, lumen; MNC, monocytes; EC, endothelial cells; N, nucleus. 
the presence of adiponectin on the luminal and basal membranes in ECs.

Figure 2 demonstrates the incorporation of adiponectin in ECs, especially in endocytic vesicles, but not in multivesicular body, lysosome, or Weibel-Palade body, which is specifically observed in $\mathrm{ECs}^{38}$. We have recently indicated that ECs do not produce adiponectin protein based on the lack of adiponectin mRNA in mouse aortic intima $^{35}$. Adiponectin is secreted from adipocytes and the secretory pathways in these cells have been reported in detail previously ${ }^{39-43}$. The presence of adiponectin in the endocytic vesicles of EC suggests that it is endocytozed from the circulation into the EC. Further studies are needed to clarify the molecular mechanism involved in endocytosis of adiponectin and its intracellular destination.

Progression of atherosclerosis involves the proliferation of resident SMCs in the intima and their migration of SMCs from the media layer. Two different SMC phenotypes have been described based on the distribution of myosin filaments and presence of large amounts of secretory molecule apparatus ${ }^{6-8}$. Local inflammatory stimuli cause phenotypic switch of SMCs from contractile type to synthetic type ${ }^{37,38,44}$. Previous in vitro experiments demonstrated that vascular SMCs produce adiponectin and SMCs-derived adiponectin keeps SMCs in contractile type in autocrine and paracrine fashions ${ }^{45}$. Interestingly, immunoelectron microscopy in the present study clearly demonstrated the presence of adiponectin protein on the surface of synthetic SMCs in ApoE-KO mice, while such signal was not detected in contractile SMCs of WT and ApoE-KO mice. Adiponectin significantly suppressed the proliferation and migration of human aortic SMCs (HASMCs) induced by platelet-derived growth factor (PDGF)-BB, basic fibroblast growth factor (bFGF), and heparin-binding epidermal growth factor-like growth factor $(\mathrm{HB}-\mathrm{EGF})^{34,46}$. The present results seem to imply that the presence of adiponectin in synthetic SMCs serves to prevent the proliferation and migration of SMCs. Further studies are needed to determine the selection mechanism of adiponectin in synthetic SMCs rather than in contractile SMCs.

Our results also showed the presence of adiponectin signal on the surface of monocytes attached to ECs (Fig. 6). At the site of atherosclerotic lesions, monocytes attached to ECs subsequently invade the atherosclerotic plaque and then transform into macrophages. Although adiponectin was detected in monocytes, it was not detected in macrophages and foam cells. Previous studies showed that adenovirus-mediated overproduction of human adiponectin in plasma (adiponectin concentration in plasma was around $500 \mu \mathrm{g} / \mathrm{mL}$ ) correlated with immunostaining for Mac-1, a marker of macrophages, in the atherosclerotic lesion of ApoE-KO mice ${ }^{26}$. However, in the present study, endogenous adiponectin protein was not detected in lipid-loaded macrophages (Fig. 4c). The discrepancy in the results of the two studies could be due to methodological differences (use of adenovirus-mediated overproduction versus gene knockout). The effect of adiponectin on macrophages has been examined using human monocyte-derived macrophages in in vitro studies ${ }^{33,47}$, but so far there is no evidence in the literature on whether endogenous adiponectin directly acts in vivo on macrophages. It is possible that adiponectin detaches from monocytes once these cells emigrate into the sub-endothelium and transform into macrophages, suggesting that adiponectin function is limited to the process of monocyte attachment to ECs.

Activated monocytes express various receptors for cytokines and chemokines $^{48-50}$, but the expression levels of adiponectin-binding molecules, such as T-cadherin ${ }^{51}$, calreticulin ${ }^{52}$, and AdipoRs ${ }^{53}$, in monocytes remains uncertain. Furthermore, it is not clear whether these molecules are up- or down-regulated upon activation of monocytes and/or their attachment to ECs. In addition, there are no data on whether adiponectin binds to monocytes in circulating peripheral blood and/or other immune cells. Further investigations are needed to understand the anti-inflammatory properties of adiponectin.
Interestingly, analysis of adiponectin in the aorta showed a shift in its localization from normal to atherosclerotic aorta. Histological analyses conducted in the present study did not delineate the mechanism responsible for such shift. While there is no evidence for differences in the expression pattern and distribution of adiponectin-binding proteins ${ }^{51-54}$ between atherosclerotic and non-atherosclerotic lesions, it is a possible that the expression levels of these adiponectin-binding proteins in the aorta may change under physiological and pathological conditions. In this context, further studies are required to determine the expression patterns of adiponectinbinding proteins in relation to the expression pattern of adiponectin in normal and atherosclerotic aortas. It is possible that yet unidentified adiponectin-binding molecules could contribute to the vascular localization of adiponectin.

Fig. 7 provides a summary of the present results. Adiponectin proteins localize in lumina and basal plasma membrane of ECs and part of adiponectin exists in the endocytic vesicles of ECs in the normal vasculature (Fig. 7, top). Adiponectin is not expressed in other components of the wall of the normal aorta. However, the localization pattern of adiponectin changes during atherosclerosis; adiponectin is expressed in ECs (mainly on luminal plasma membrane), synthetic SMCs, and monocytes in the atherosclerotic vasculature (Fig. 7, bottom). We conclude that analysis of ultrastructural changes in adiponectin localization may provide novel insights into the pathogenesis of atherosclerosis.

\section{Methods}

Animals. Male apolipoprotein E knockout (ApoE-KO) mice were obtained from Charles River Japan Inc. (Kanagawa, Japan). Adiponectin knockout (Adipo-KO) mice were generated and backcrossed as described previously ${ }^{24}$. Both ApoE-KO and Adipo-KO mice were of C57BL/6J background and thus C57BL/6J mice were used as wild-type (WT) mice. Mice were maintained at $22^{\circ} \mathrm{C}$ under a $12: 12$-h light-dark cycle (lights on from 8:00 to 20:00). For analysis of immunohistochemistry, male WT and Adipo-KO mice were used at 10 weeks of age. For atherosclerosis model mice, male ApoE-KO mice were fed high-fat atherogenic western diet (WTD, Oriental Yeast; $20 \%$ fat, $0.15 \%$ cholesterol) from 8 to 20 weeks of age. Mice were anesthetized by intraperitoneal injection of medetomidine $(0.3 \mathrm{mg} / \mathrm{kg}$ body weight $)$, midazolam ( $4 \mathrm{mg} / \mathrm{kg}$ body weight), and butorphanol ( $5 \mathrm{mg} / \mathrm{kg}$ body weight), and then the thoracic ascending aorta was excised. To eliminate contamination by circulating adiponectin, mice were transcardially perfused with cold saline before the collection of aorta. The experimental protocol was approved by the Ethics Review Committee for Animal Experimentation of Osaka University School of Medicine. This study also conforms to the Guide for the Care and Use of Laboratory Animals published by the US National Institutes of Health.

Immunohistochemistry. Following the dissection, the aortas were thoroughly flushed several times with ice-cold saline, and subsequently embedded and frozen in Tissue-Tek O.C.T. Compound (Sakura Finetek, Torrance, CA). Frozen aorta tissue specimens were cut at $6 \mu \mathrm{m}$-thick-sections on a cryostat, fixed in ice-cold acetone for $20 \mathrm{~min}$ and washed for $5 \mathrm{~min}$ in phosphate buffered saline (PBS) ( $\mathrm{pH} \mathrm{7.4).} \mathrm{Sections}$ were incubated with blocking buffer (Protein Block Serum-Free, Dako, CA) for $10 \mathrm{~min}$ at room temperature, and then incubated overnight with goat antiadiponectin (dilution, $1: 100$; R\&D systems Inc., Minneapolis, MN) or control goat IgG in PBS containing $1 \%$ fetal bovine serum (FBS). Labeling was visualized by biotinylated anti-goat IgG antibody ( $1: 200$; Vector Laboratories, Burlingame, CA) and avidin-biotin-horseradish peroxidase procedure (Vectastatin ABC kit, Vector laboratories) with 3,3'-diaminobenzidine tetrahydrochloride (DAB, Sigma-Aldrich, St Louis, MO) according to the protocol recommended by the manufacturer. To confirm atherosclerotic plaque formation, sections from ApoE-KO mice were stained with hematoxylin and eosin ( $\mathrm{H} \& \mathrm{E}$ ) or Oil Red O. Light microscopy was carried out with a Provis AX-80 microscope (Olympus, Tokyo, Japan).

Immunofluorescence staining. Double immunofluorescence staining was performed as described previously ${ }^{35}$. Sections were incubated with goat antiadiponectin $(1: 100)$, and rat anti-CD31 ( $1: 100$; BD Biosciences, San Jose, CA), or rabbit anti- $\alpha$-SMA $(1: 200$, Abcam Inc., Cambridge, MA) or rat anti-Moma- $2(1: 50$, Abcam) antibody in PBS containing $1 \%$ FBS. To confirm the specificity of antibody for adiponectin, the antibody absorption test was performed by combining the primary antibody with a 20 -fold molar excess of synthetic peptide of adiponectin (R\&D systems) followed by incubation overnight at $4{ }^{\circ} \mathrm{C}$, before application to tissue sections. Donkey anti-goat IgG conjugated Alexa 488 (Life Technologies,

Gaithersburg, MD), chicken anti-rabbit IgG conjugated Alexa 594 (Life Technologies), or chicken anti-rat IgG conjugated Alexa 594 were used as the secondary antibody. Cell nuclei were counterstained with DAPI. Microscopy was 

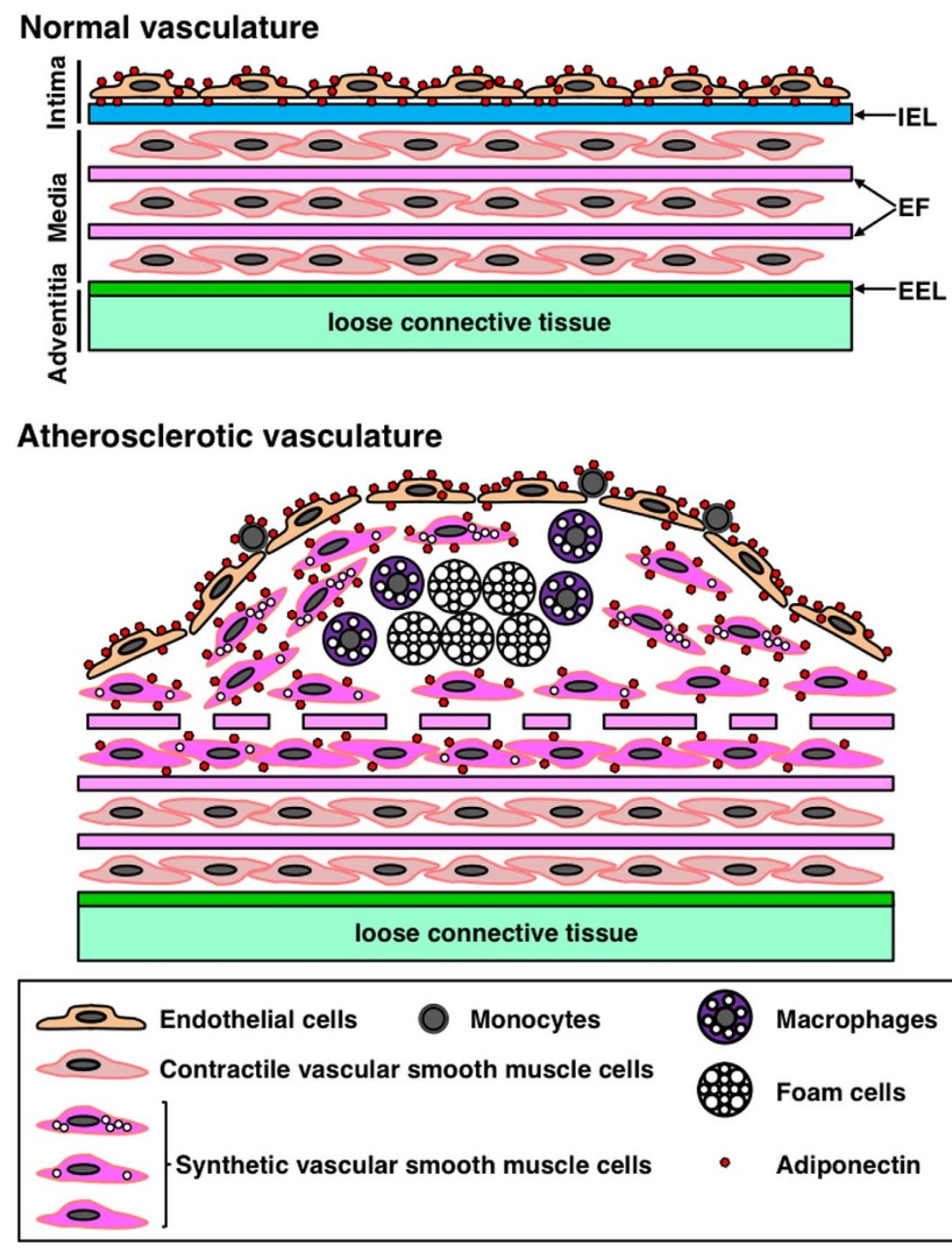

Figure $7 \mid$ Schematic diagram illustrating the localization of adiponectin in normal and atherosclerotic aortas. In the aorta of WT mice (top), adiponectin is localized in lumina and basal plasma membrane of endothelial cells (ECs). Part of adiponectin is present in endocytic vesicles of ECs. In atherosclerotic lesions of ApoE-KO mice (bottom), adiponectin is present in ECs, synthetic smooth muscle cells (SMCs), and monocytes. Adiponectin is mainly localized on luminal plasma membrane of ECs. In synthetic SMCs and monocytes, adiponectin is localized on the cell surface membrane. IEL, internal elastic lamina; EF, elastic fiber; EEL, external elastic fiber.

performed using an Olympus FV1000D confocal laser scanning microscope system (Olympus).

Immunoelectron microscopy. Following dissection of the aorta, the aortas were thoroughly flushed with several changes of ice-cold saline, and subsequently fixed overnight in $0.1 \mathrm{M}$ PBS containing $3 \%$ paraformaldehyde and $0.15 \%$ glutaraldehyde (GA). After washing with $0.1 \mathrm{M}$ PBS, the tissues were immersed in $6 \%$ gelatin and then $12 \%$ gelatin solution. Following stiffening the gelatin by refrigeration, the samples were cut into $40 \mu \mathrm{m}$ thick sections using vibrating blade microtome (VT1000s; Leica, Wetzlar, Germany). To detect adiponectin in the aorta, the sections were immunostained by the pre-embedding immunoperoxidase technique and the preembedding immunogold labeling technique. In the first method, similar to the immunoperoxidase method described above, the harvested tissues treated with goat anti-adiponectin antibody were developed with $\mathrm{DAB}$, and then rinsed with $0.1 \mathrm{MPB}$. For pre-embedding immunogold labeling, the tissue sections were first treated with goat anti-adiponectin primary antibody $(1: 100)$, then incubated overnight at $4{ }^{\circ} \mathrm{C}$ with gold-conjugated rabbit anti-goat IgG secondary antibody $(1: 500$; Nanoprobes, Yaphank, NY) in $0.1 \mathrm{M}$ PBS containing $1 \%$ FBS. After thorough washing with $0.1 \mathrm{M}$ phosphate buffer (PB), the sections were refixed in $0.1 \mathrm{M}$ PB containing $1 \%$ GA and then treated with $0.1 \mathrm{M} \mathrm{PB}$ containing $50 \mathrm{mM}$ glycine to inhibit nonspecific binding to aldehyde groups. To amplify signals to a detectable level, gold enhancement was performed according to the instructions provided by the manufacturer

(Nanoprobes). After washing in distilled water, the samples were immersed in $0.1 \mathrm{M}$ PB. Finally, the samples immunostained using both methods were prepared for electron microscopy as described previously ${ }^{55}$. All electron micrographs were obtained using transmission electron microscope (H-7650; Hitachi, Tokyo).

1. Aschoff, L. Observations concerning the relationship between cholesterol metabolism and vascular disease. Br. Med. J. 2, 1131-1134 (1932).

2. Kao, V. C. \& Wissler, R. W. A study of the immunohistochemical localization of serum lipoproteins and other plasma proteins in human atherosclerotic lesions. Exp. Mol. Pathol. 4, 465-479 (1965).

3. Walton, K. W. \& Williamson, N. Histological and immunofluorescent studies on the evolution of the human atheromatous plaque. J. Atheroscler. Res. 8, 599-624 (1968).

4. Ylä-Herttuala, S. et al. Evidence for the presence of oxidatively modified low density lipoprotein in atherosclerotic lesions of rabbit and man. J. Clin. Invest. 84, 1086-1095 (1989).

5. Hollander, W., Colombo, M. A., Kirkpatrick, B. \& Paddock, J. Soluble proteins in the human atherosclerotic plaque. With spectral reference to immunoglobulins, C3-complement component, alpha 1-antitrypsin and alpha 2-macroglobulin. Atherosclerosis 34, 391-405 (1979).

6. Ross, R. The pathogenesis of atherosclerosis: a perspective for the 1990s. Nature 362, 801-809 (1993).

7. Ross, R. Atherosclerosis-an inflammatory disease. N. Engl. J. Med. 340, 115-126 (1999).

8. Libby, P. Inflammation in atherosclerosis. Nature 420, 868-874 (2002). 
9. Matsuzawa, Y. Therapy Insight: adipocytokines in metabolic syndrome and related cardiovascular disease. Nat. Clin. Pract. Cardiovasc. Med. 3, 35-42 (2006).

10. Matsuzawa, Y., Funahashi, T., Kihara, S. \& Shimomura, I. Adiponectin and metabolic syndrome. Arterioscler. Thromb. Vasc. Biol. 24, 29-33 (2004).

11. Okubo, K. et al. Large scale cDNA sequencing for analysis of quantitative and qualitative aspects of gene expression. Nat. Genet. 2, 173-179 (1992).

12. Maeda, K. et al. Analysis of an expression profile of genes in the human adipose tissue. Gene 190, 227-235 (1997).

13. Maeda, K. et al. cDNA cloning and expression of a novel adipose specific collagenlike factor, apM1 (AdiPose Most abundant Gene transcript 1). Biochem. Biophys. Res. Commun. 221, 286-289 (1996).

14. Nakano, Y., Tobe, T., Choi-Miura, N. H., Mazda, T. \& Tomita, M. Isolation and characterization of GBP28, a novel gelatin-binding protein purified from human plasma. J. Biochem. 120, 803-812 (1996).

15. Scherer, P. E., Williams, S., Fogliano, M., Baldini, G. \& Lodish, H. F. A novel serum protein similar to C1q, produced exclusively in adipocytes. J. Biol. Chem. 270, 26746-26749 (1995).

16. Hu, E., Liang, P. \& Spiegelman, B. M. AdipoQ is a novel adipose-specific gene dysregulated in obesity. J. Biol. Chem. 271, 10697-10703 (1996).

17. Arita, Y. et al. Paradoxical decrease of an adipose-specific protein, adiponectin, in obesity. Biochem. Biophys. Res. Commun. 257, 79-83 (1999).

18. Kumada, M. et al. Association of hypoadiponectinemia with coronary artery disease in men. Arterioscler. Thromb. Vasc. Biol. 23, 85-89 (2003).

19. Pischon, T. et al. Plasma adiponectin levels and risk of myocardial infarction in men. JAMA 291, 1730-1737 (2004).

20. Hotta, K. et al. Plasma concentrations of a novel, adipose-specific protein, adiponectin, in type 2 diabetic patients. Arterioscler. Thromb. Vasc. Biol. 20 , 1595-1599 (2000)

21. Lindsay, R. S. et al. Adiponectin and development of type 2 diabetes in the Pima Indian population. Lancet 360, 57-58 (2002).

22. Spranger, J. et al. Adiponectin and protection against type 2 diabetes mellitus. Lancet 361, 226-228 (2003).

23. Fruebis, J. et al. Proteolytic cleavage product of $30-\mathrm{kDa}$ adipocyte complementrelated protein increases fatty acid oxidation in muscle and causes weight loss in mice. Proc. Natl. Acad. Sci. U. S. A. 98, 2005-2010 (2001).

24. Maeda, N. et al. Diet-induced insulin resistance in mice lacking adiponectin/ ACRP30. Nat. Med. 8, 731-737 (2002).

25. Kubota, N. et al. Disruption of adiponectin causes insulin resistance and neointimal formation. J. Biol. Chem. 277, 25863-25866 (2002).

26. Okamoto, Y. et al. Adiponectin reduces atherosclerosis in apolipoprotein Edeficient mice. Circulation 106, 2767-2770 (2002).

27. Matsuda, M. et al. Role of adiponectin in preventing vascular stenosis. The missing link of adipo-vascular axis. J. Biol. Chem. 277, 37487-37491 (2002).

28. Yokota, T. et al. Adiponectin, a new member of the family of soluble defense collagens, negatively regulates the growth of myelomonocytic progenitors and the functions of macrophages. Blood 96, 1723-1732 (2000).

29. Kamada, Y. et al. Enhanced carbon tetrachloride-induced liver fibrosis in mice lacking adiponectin. Gastroenterology 125, 1796-1807 (2003).

30. Fujita, K. et al. Adiponectin protects against angiotensin II-induced cardiac fibrosis through activation of PPAR-alpha. Arterioscler. Thromb. Vasc. Biol. 28, 863-870 (2008).

31. Ouchi, N. et al. Novel modulator for endothelial adhesion molecules: adipocytederived plasma protein adiponectin. Circulation 100, 2473-2476 (1999).

32. Ouchi, N. et al. Adiponectin, an adipocyte-derived plasma protein, inhibits endothelial NF-kappaB signaling through a cAMP-dependent pathway. Circulation 102, 1296-1301 (2000).

33. Ouchi, N. et al. Adipocyte-derived plasma protein, adiponectin, suppresses lipid accumulation and class A scavenger receptor expression in human monocytederived macrophages. Circulation 103, 1057-1063 (2001).

34. Arita, Y. et al. Adipocyte-derived plasma protein adiponectin acts as a plateletderived growth factor-BB-binding protein and regulates growth factor-induced common postreceptor signal in vascular smooth muscle cell. Circulation $\mathbf{1 0 5}$, 2893-2898 (2002)

35. Komura, N. et al. Adiponectin protein exists in aortic endothelial cells. PLoS One 8, e71271 (2013)

36. Ross, R. \& Glomset, J. A. Atherosclerosis and the arterial smooth muscle cell: Proliferation of smooth muscle is a key event in the genesis of the lesions of atherosclerosis. Science 180, 1332-1339 (1973).

37. Libby, P., Ridker, P. M. \& Hansson, G. K. Progress and challenges in translating the biology of atherosclerosis. Nature 473, 317-325 (2011).

38. Weibel, E. R. \& Palade, G. E. New cytoplasmic components in arterial endothelia. J. Cell. Biol. 23, 101-112 (1964).

39. Bogan, J. S. \& Lodish, H. F. Two compartments for insulin-stimulated exocytosis in 3T3-L1 adipocytes defined by endogenous ACRP30 and GLUT4. J. Cell. Biol. 146, 609-620 (1999)

40. Bose, A. et al. The v-SNARE Vtila regulates insulin-stimulated glucose transport and Acrp30 secretion in 3T3-L1 adipocytes. J. Biol. Chem. 280, 36946-36951 (2005)
41. Wang, Z. V.et al. Secretion of the adipocyte-specific secretory protein adiponectin critically depends on thiol-mediated protein retention. Mol. Cell. Biol. 27, 3716-3731 (2007).

42. Qiang, L., Wang, H. \& Farmer, S. R. Adiponectin secretion is regulated by SIRT1 and the endoplasmic reticulum oxidoreductase Ero1-L alpha. Mol. Cell. Biol. 27, 4698-4707 (2007).

43. Wang, Z. V. \& Scherer, P. E. DsbA-L is a versatile player in adiponectin secretion. Proc. Natl. Acad. Sci. U. S. A. 105, 18077-18078 (2008).

44. Owens, G. K., Kumar, M. S. \& Wamhoff, B. R. Molecular regulation of vascular smooth muscle cell differentiation in development and disease. Physiol. Rev. 84, 767-801 (2004).

45. Ding, M. et al. Vascular smooth muscle cell-derived adiponectin: a paracrine regulator of contractile phenotype. J. Mol. Cell. Cardiol. 52, 474-484 (2012).

46. Wang, Y. et al. Adiponectin inhibits cell proliferation by interacting with several growth factors in an oligomerization-dependent manner. J. Biol. Chem. 280, 18341-18347 (2005).

47. Kumada, M. et al. Adiponectin specifically increased tissue inhibitor of metalloproteinase-1 through interleukin-10 expression in human macrophages Circulation 109, 2046-2049 (2004).

48. Loppnow, H., Buerke, M., Werdan, K. \& Rose-John, S. Contribution of vascular cell-derived cytokines to innate and inflammatory pathways in atherogenesis. J. Cell. Mol. Med. 15, 484-500 (2011).

49. Ingersoll, M. A., Platt, A. M., Potteaux, S. \& Randolph, G. J. Monocyte trafficking in acute and chronic inflammation. Trends. Immunol. 32, 470-477 (2011).

50. White, G. E., Iqbal, A. J. \& Greaves, D. R. CC chemokine receptors and chronic inflammation--therapeutic opportunities and pharmacological challenges. Pharmacol. Rev. 65, 47-89 (2013).

51. Hug, C. et al. T-cadherin is a receptor for hexameric and high-molecular-weight forms of Acrp30/adiponectin. Proc. Natl. Acad. Sci. U. S. A. 101, 10308-10313 (2004).

52. Takemura, Y. et al. Adiponectin modulates inflammatory reactions via calreticulin receptor-dependent clearance of early apoptotic bodies. J. Clin. Invest. 117, 375-386 (2007).

53. Yamauchi, T. et al. Cloning of adiponectin receptors that mediate antidiabetic metabolic effects. Nature 423, 762-769 (2003)

54. Okamoto, Y. et al. An adipocyte-derived plasma protein, adiponectin, adheres to injured vascular walls. Horm. Metab. Res. 32, 47-50 (2000).

55. Koyama, Y., Nishida, T. \& Tohyama, M. Establishment of an optimised protocol for a Golgi-electron microscopy method based on a Golgi-Cox staining procedure with a commercial kit. J. Neurosci. Methods. 218, 103-109 (2013).

\section{Acknowledgments}

We thank the Center of Medical Research and Education, Graduate School of Medicine, Osaka University, for the excellent technical support. We also thank all members of the III ${ }^{\text {rd }}$ laboratory (Adiposcience laboratory), Department of Metabolic Medicine, Osaka University, for the helpful discussion on the project. This work was supported in part by a Grant-in-Aid for Scientific Research (C) no. 22590979 (to N.M.), a Grant-in-Aid for Scientific Research (B) no. 24390238 (to I.S.), a Grant-in-Aid for Scientific Research on Innovative Areas no. 22126008 (to T.F.), and Takeda Science Foundation (to N.M.). The funding agencies had no role in study design, data collection and analysis, decision to publish, or preparation of the manuscript.

\section{Author contributions}

N.M., Y.N., T.F., S.S. and I.S. designed the project; T.M., Y.K., Y.N., Y.F. and K.M. performed research; T.M., Y.K., N.M. and Y.N. analyzed data; T.M., Y.K., N.M. and Y.N wrote the paper. T.F., S.S. and I.S. edited the manuscript.

\section{Additional information}

Supplementary information accompanies this paper at http://www.nature.com/ scientificreports

Competing financial interests: The authors declare no competing financial interests. How to cite this article: Mori, T. et al. Ultrastructural Localization of Adiponectin protein in Vasculature of Normal and Atherosclerotic mice. Sci. Rep. 4, 4895; DOI:10.1038/ srep04895 (2014)

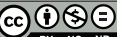

This work is licensed under a Creative Commons Attribution-NonCommercialNoDerivs 3.0 Unported License. The images in this article are included in the article's Creative Commons license, unless indicated otherwise in the image credit; if the image is not included under the Creative Commons license, users will need to obtain permission from the license holder in order to reproduce the image. To view a copy of this license, visit http://creativecommons.org/licenses/by-nc-nd/3.0/ 